\title{
LES PIONNIERS DE LA PROTECTION CONTRE LES RAYONNEMENTS IONISANTS*
}

\author{
A. LACASSAGNE** \\ (Manuscrit reçu le 25 novembre 1968 )
}

Aucune découverte scientifique ne suscita une exaltation aussi intense et rapidement répandue que celle des rayons $\mathrm{X}$, aucune ne fut suivie d'applications aussi immédiates.

Le 28 décembre 1895 , le professeur de Physique Conrad RöNTGEN faisait connaître, à une réunion de la Société physico-médicale de Würzburg, l'existence d'un rayonnement nouveau, produit sous l'action de décharges électriques dans un tube à air raréfié. Ces rayons invisibles possédaient des propriétés extraordinaires, ils traversaient les corps solides qui se révélaient plus ou moins opaques. C'est ainsi que, sur un écran de platino-cyanure de baryum qui devenait fluorescent, on voyait apparaître le squelette de sa main lorsqu'on interposait celle-ci entre le tube émetteur et l'écran. Dans le gros livre, paru en 1965 sous le titre évocateur "The trail of the invisible light ", où le Dr. Grigg de Chicago a collecté les moindres détails relatifs à l'origine et au développement de la découverte des rayons $\mathrm{X}$ et de celle du radium, il est rapporté que la première radiographie d'une main, où se voyaient distinctement tous les os et notamment une phalange encerclée de l'alliance, aurait été prise sur Mme RöNTGEN, le 8 novembre r895.

L'émotion produite par ces révélations fut intense; l'Homme allait désormais disposer du privilège que la superstition des Anciens attribuait à l'œil du lynx, de voir à travers les murailles. La grande presse s'empara aussitôt de la nouvelle et la répandit dans le monde entier. Les médecins et particulièrement les électrologistes et les chirurgiens entrevirent les immenses services que ces techniques allaient leur rendre; des industriels se mirent à rivaliser pour la préparation du matériel et la construction d'appareils pratiques. Des esprits curieux et novateurs, aussi bien parmi les photographes que parmi les médecins, allaient se consacrer à cette nouvelle spécialité. Dès le mois de janvier 1896 commençaient à paraître, dans les journaux de médecine et de physique, les articles de ceux qui avaient vérifié les faits annoncés par RöNTGEN; il en fut publié un millier dans le courant de la seule année I896; sans compter les articles de ceux qui revendiquaient la priorité de la découverte.

* Conférence prononcée au cours du I er Congrès Européen de Radioprotection à Menton le 9 octobre r 968 . Ce texte sera publié dans le compte rendu du Congrès actuellement sous presse.

** Professeur Lacassagne, ex-Directeur de la section médicale et biologique de l'Institut du Radium, Professeur honoraire au Collège de France, Membre de l'Institut, Président de la Ligue nationale française contre le cancer. 
Parmi les premières radiographies de cas cliniques, on peut citer celle faite à Liverpool par le physicien LODGE, qui avait repéré la balle incluse dans le poignet d'un blessé du Royal Southern Hospital; et celle relatée dans une note de LANNELONGUE, BARTHELEMY et OUdin, intitulée « De l'utilité de la photographie aux rayons $\mathrm{X}$ dans la pathologie humaine "), qu'Henri PorNCARÉ présenta le 20 janvier à l'Académie des Sciences; les clichés faits à l'Hôpital Trousseau par Oudin et BARTHELEMY avaient permis au chirurgien LANNELONGUE de diagnostiquer l'extension, à l'articulation, de la lésion tuberculeuse d'une phalange.

Cependant, la nature du nouveau rayonnement restait mystérieuse. RöNTGEN, dans sa communication, avait énuméré que celui-ci n'était, ni infrarouge, ni visible, ni ultraviolet, ni cathodique; l'utilisation de la lettre $\mathrm{X}$ pour le désigner était un aveu d'ignorance. Les grands maîtres de la physique en discutaient, et je veux rappeler ici l'article d'Henri PornCARÉ, paru à ce sujet dans le numéro du 30 janvier 1896 de la " Revue Générale des Sciences », qui suggéra à Henri BECQUEREL sa fameuse expérience révélatrice de la radioactivité naturelle.

I. - Evidemment tous ces essayeurs, qui produisaient et utilisaient les rayons $\mathrm{X}$, en recevaient eux-mêmes sans méfiance. Rien ne permettait de supposer a priori une action maléfique de ces précieux agents. Mais, déjà en mars 1896 , des lettres aux éditeurs de journaux scientifiques des Etats-Unis signalaient des faits révélateurs d'une action biologique du rayonnement de Röntgen. Le premier effet indiscutable, rapporté par DANIEL, fut celui d'une dépilation locale survenue une vingtaine de jours après la longue recherche d'un projectile crânien; quelques mois plus tard, en juillet 1896 , MARCuSE rapportait l'observation d'une " dermatite » apparue chez un garçon de 17 ans qui, depuis quatre semaines, une ou deux fois par jour et pendant s ou ro minutes, servait de sujet pour la démonstration commerciale de la transillumination des poumons et du cœur au moyen des rayons $\mathrm{X}$.

Et tout de suite, ceux qu'on peut désigner comme les premiers röntgenologistes se divisèrent en deux groupes psychologiquement opposés : les confirmatifs et les négateurs.

Parmi ces derniers figurent d'abord ceux qui prétendaient que le nouveau rayonnement n'était pas responsable de ces méfaits, et les attribuaient à d'autres causes : décharges électrostatiques, courants induits, ultraviolet, ozone, etc... En I901 encore, de tels points de vue étaient soutenus par certains. D'autres, sans nier les altérations qu'au cours des années les expérimentateurs constataient dans des organes irradiés, en minimisaient les dangers et critiquaient les mesures de protection - excessives à leur gré - que préconisaient les röntgenologistes de l'autre groupe. On devait entendre resurgir les mêmes arguments, 35 ans plus tard, lorsque cette double mentalité se manifesta parmi les médecins, à propos de l'emploi du thorotrast.

De leur côté, les pionniers de la protection recherchaient les moyens de préserver les sujets à exposer, et eux-mêmes, tout en utilisant au mieux les incomparables instruments de recherche et de thérapie que leur avaient fournis RöNTGEN d'abord, Pierre et Marie CuRIE ensuite. Ils reprenaient, pour leur compte et sur un terrain plus généreux, la lutte sans cesse renouvelée entre le projectile et la cuirasse. 
Le Docteur Elihu Thomson, voulant apporter la preuve d'une action caustique des nouveaux rayons, soumit à une irradiation l'auriculaire de sa main gauche; il décrivit les symptomes et l'évolution de la « Roentgen ray burn » ainsi provoquée par l'absorption, dans la peau, du rayonnement émis par un " tube mou ". Afin de répondre à des objections il refit l'expérience sur un autre de ses doigts; mais celui-ci était entouré de plomb, sauf au niveau d'une petite fenêtre recouverte seulement d'une mince feuille d'aluminium : la radiolésion ne se manifesta qu'en dessous de cet espace non protégé.

Un dentiste, William Rollins avait été un des premiers à être passionné, en Amérique, par la découverte de RöNTGEN. Il construisit lui-même, avec ingéniosité, ses appareillages. Un des premiers, aussi, il admit sans réserve le danger que les rayons $\mathrm{X}$ pourraient faire courir. Pendant des années, il s'efforça de préciser et de faire connaître des moyens de protection : lunettes en verre au plomb; écrans; tube enfermé dans une enveloppe absorbante ménageant seulement une ouverture restant obturée par un diaphragme pendant tout le temps de la mise en place correcte du dispositif; protection du patient par un revêtement peu perméable aux rayons et ne laissant découvert que le champ destiné à recevoir le plus petit cône de rayons et pendant le moins de temps possible. En effet, ayant eu la curiosité, dès 1898 , d'irradier des cobayes, avec comme résultat leur mort en quelques jours, il était convaincu de la nocivité générale des rayons $\mathrm{X}$ pour l'ensemble de l'organisme.

En France, le Dr Antoine BÉclère, médecin des Hôpitaux de Paris, fut le premier à spécialiser son service, en 1897 , à l'étude de la radiologie. Il se fit aussi le protagoniste des " moyens de protection du médecin et des malades contre l'action nocive des nouvelles radiations : rayons de Röntgen et rayons du radium ). Il en précisa les règles, dans un article paru sous ce titre en 1904. Au cours des visites qu'il avait faites à l'étranger, en Allemagne et en Autriche où les recherches aussi bien expérimentales que cliniques au moyen du rayonnement Röntgen avaient pris un grand essor, il avait été particulièrement frappé (à Hambourg), par les précautions en vigueur dans le laboratoire d'AlBers-SchönBERg.

Comme on le sait, c'est à ce dernier que l'on doit la découverte publiée en 1903, de l'action stérilisante des rayons $\mathrm{X}$ sur le testicule. Dans son service, l'opérateur s'enfermait dans une petite cabine amovible, revêtue de lames de plomb, de l'intérieur de laquelle il pouvait surveiller le sujet exposé (homme ou animal) à travers un regard en verre au plomb, et manipuler toutes les commandes. Pour la radioscopie, on devait se contenter de paravents doublés de plomb protégeant, des aisselles jusqu'aux pieds, le radiologiste muni de gants enduits de sous-nitrate de bismuth, de lunettes ad hoc et de tabliers protecteurs.

Mis au courant de ces dispositifs, un brillant jeune radiologiste de Paris déclara aussitôt que, en France, les malades n'accepteraient jamais que le médecin restât éloigné d'eux pendant les applications radiothérapiques !

C'est en 1909 que je fus initié à l'emploi des rayons X, au laboratoire d'histologie de la faculté de médecine de Lyon. En seconde année d'internat, ayant opté pour une carrière de recherche scientifique j'y étais venu demander un sujet de thèse au Dr Regaud, alors professeur agrégé et chef des travaux. Depuis 1906, cet histologiste étudiait, avec ses collaborateurs, l'action du rayonnement de Röntgen sur différents tissus et organes. Je fus chargé d'aborder les effets de l'irradiation sur l'ovaire. 
Mon maître, suivant les conseils du Dr Nogrer, professeur agrégé de physique médicale, avait aménagé une vaste salle du laboratoire en chambre d'irradiation. L'appareillage et la technique étaient sans cesse améliorés; ce qui n'empêchait pas les pannes assez fréquentes, ne serait-ce que la nécessité du nettoyage malodorant du mercure de l'interrupteur, ou le retard de l'arrivée de nouvelles ampoules commandées en Allemagne. Et cependant, nous étions plusieurs travailleurs attendant impatiemment que notre tour vienne d'obtenir une de ces séances d'irradiation, à l'installation et à la clôture desquelles M. REGAud présidait toujours. Sous l'ampoule se succédèrent, selon l'époque: rats, chats, lapins, œufs d'ascaris, têtards de grenouilles, chiens, béliers et même quelques cancéreux inopérables.

Le Dr Nogrer insistait sur les deux moyens de protection qu'on devait combiner selon les cas : $\mathrm{I}^{\circ}$ ) arrêter les rayons à la source même et les empêcher de passer partout où ils ne sont pas utilisés ; $2^{\circ}$ ) laisser l'ampoule émettre des rayons dans tous les sens, mais protéger l'opérateur et l'opéré « en les mettant à l'ombre ". En réalité, pour nous autres, les élèves, qui ne séjournions occasionnellement dans la salle des rayons $\mathrm{X}$ que les seuls jours où il nous était permis de faire une expérience, ces précautions étaient réduites à leur plus simple expression : la distance. Il nous était recommandé de surveiller l'opération du fond de la pièce, et de nous approcher de l'ampoule enfermée dans son manchon de caoutchouc au plomb que pour la "ramollir» éventuellement en chauffant l'osmorégulateur et pour vérifier de temps en temps, au moyen d'une pastille de platino-cyanure de baryum fixée à l'extrémité d'une longue planchette en bois, que l'animal n'avait pas bougé et que le faisceau recouvrait bien le champ à traiter. Mais nous ne disposions ni de lunettes, ni de gants, ni de tablier.

Et cependant, d'Hallurn prouvait la difficulté de se protéger pendant les séances de röntgenthérapie. Il avait vérifié, au moyen de plaques photographiques, que l'opérateur situé en face du tableau de réglage, dans une pièce voisine de la salle de traitement ne communiquant avec celle-ci que par une porte entrouverte, recevait pourtant des rayons X, les uns émis par les soupapes, les autres traversant localisateur et mur de protection, ainsi que des rayons secondaires émis par le plafond et les murs.

Mais la protection du radiodiagnosticien, qui avait posé des problèmes encore plus difficiles à résoudre, se présenta, peu après le début de la guerre de I914, avec un caractère d'urgente gravité. Dès les premiers jours des grandes offensives, une brusque affluence de blessés se produisit dans des formations hospitalières où les appareillages et le personnel qualifié pour la recherche des projectiles étaient très insuffisants. L'an dernier, la célébration du centenaire de la naissance de Marie Curie a été l'occasion de rappeler le rôle important que celleci joua en France, lorsqu'elle se consacra immédiatement à l'installation de postes de radiodiagnostic dans les hôpitaux du territoire, et à l'enseignement d'infirmières manipulatrices. Elle fit partie, avec les docteurs BÉCLÈre et REGAUD, du Comité consultatif de radiologie et de celui des corps radioactifs lorsqu'ils furent enfin créés officiellement. Il importait de tout mettre en œuvre pour que les nombreux médecins radiologistes hâtivement formés, et qui seraient inévitablement et longuement exposés aux rayons $\mathrm{X}$ pendant les périodes de fonctionnement intensif dans les formations sanitaires de l'armée, sachent et puissent appliquer au maximum les précautions de défense. 
II. - En I919, la nécessité d'une réorganisation générale de chacune des anciennes nations belligérantes ouvrit une nouvelle période de l'histoire de la protection contre les radiations ionisantes. Et ceci, dans deux directions : d'une part, l'emploi thérapeutique des corps radioactifs, auquel Mme Curie et le docteur REgAud donnèrent une vigoureuse impulsion en ouvrant enfin à la recherche le nouvel Institut du Radium de l'Université de Paris; d'autre part, la règlementation des principes fondamentaux de la protection du personnel et des malades dans l'emploi des rayons $\mathrm{X}$, dont le pouvoir de pénétration et les indications allaient sans cesse s'accroître; des radiologistes britanniques en prirent l'initiative.

M. Regaud, homme des réalisations hardies, entendait entreprendre sans plus de retard la curiethérapie des cancers, afin de mettre en application les conceptions que ses recherches antérieures de radiobiologie lui avaient suggérées. La mobilisation et la guerre l'avaient empêché de le faire, près de cinq ans auparavant, dans son nouveau laboratoire de l'Institut du Radium, alors qu'il venait d'en prendre la direction. A défaut de radium, difficile à se procurer au début de 1919, il disposait du radon que Mme Curie mettait largement à sa disposition. Mais c'était jouer la difficulté en ce qui concernait la protection, car l'emploi de cette émanation compliquait singulièrement les manipulations et - partant augmentait les dangers d'exposition au cours de l'extraction du gaz, de sa mise en tubes scellés, des mesures de l'activité de ceux-ci, de leur conditionnement sous forme de foyers convenant pour les traitements ou l'expérimentation. De plus, le nouveau personnel de médecins et de techniciens recruté par le directeur ignorait tout de la radioactivité. Aussi, des dispositifs de protection appropriés à ces différentes opérations furent immédiatement imaginés et mis en service : tables de manipulation protégeant la plus grande partie du corps, pinces variées pour la préhension des tubes nus, leur mise en étui-filtre, ou en aiguille, boîtes de transport d'un milieu à un autre, etc... Un hématologiste avait été engagé pour l'étude des effets de l'irradiation des organes sanguiformateurs et la surveillance de la formule du personnel. Enfin, dans le service de röntgenthérapie de la Fondation Curie qui fut construit en 1920, une protection efficace et relativement économique était assurée par des murs de refend à l'intérieur desquels avait été aménagé un intervalle de 2 à $4 \mathrm{~cm}$ que l'on comblait par du minerai de plomb pulvérisé.

A cette même époque, se développait très rapidement une industrie de préparation de corps radioactifs destinés à des usages variés. Dès 1921 , le docteur André Broca, professeur de physique avait alerté l'académie de médecine de la menace d'accidents auxquels des travailleurs de tous ordres pouvaient y être exposés.

En février 1925 , un nouveau cri d'alarme fut poussé devant cette même académie, par une commission composée de d'Arsonval, Antoine Béclère, Broca, Mme Curie, et dont le rapporteur fut M. Regaud. La Compagnie en vota les conclusions affirmant la nécessité d'un contrôle et d'une réglementation des établissements industriels et commerciaux qui préparaient, manipulaient ou transportaient des corps radioactifs; elle demanda aux Ministres de la Santé et du Travail de faire assurer ce contrôle et cette règlementation. 
III. - Un esprit collectif pour l'organisation de la protection contre les radiations ionisantes s'était manifesté très tôt en Grande-Bretagne; un comité avait été chargé par la Röntgen Society, en 1968, d'étudier les moyens d'éviter leurs effets dangereux. Mais les impératifs professionnels pendant la guerre de r914-1 8 avaient considérablement accru les risques. Un comité, dans lequel figuraient notamment le physicien Georges KAYE et le professeur de radiologie Sydney Russ, avait été chargé par le War Office de le conseiller sur l'emploi des rayons X dans l'armée. Ces pionniers de la protection se retrouvèrent peu après la fin de la guerre, dans le British Radium and X-Ray Protection Committee, créé en I92 I à l'initiative du radiologiste Stanley Melville. On y accomplit rapidement un remarquable travail de fixation de différentes mesures à prendre pour éliminer tout risque important provenant des sources de rayonnements ionisants. Rédigées sous forme de recommandations, ces règles furent bientôt généralement appliquées en Grande-Bretagne.

Présentées au $\mathrm{I}^{\mathrm{er}}$ congrès international de radiologie qui se tint à Londres en 1925 , elles y furent accueillies avec faveur. Un comité préparatoire de la protection fut alors chargé d'étudier, avec KAYE comme président et Melville comme secrétaire, les meilleures conditions permettant de protéger les travailleurs, et devant être proposées au $2^{\mathrm{e}}$ congrès international prévu pour 1928 à Stockholm. Les recommandations qui y furent présentées étaient évidemment conformes à celles du British Radium and X-Ray Protection Committee. Elles furent adoptées presque sans modification, et devinrent règles internationales. La décision de création d'un comité international de protection fut prise. Son premier bureau était composé du professeur Sievert de Stockholm comme président, KaYe et MELVILle comme secrétaires.

Depuis lors, ce comité - qui s'est progressivement renouvelé en faisant appel aux spécialistes les plus qualifiés - n'a pas cessé de travailler activement. A chacun des Congrès internationaux de Radiologie, il présente le résultat de son mandat périodique, sous forme d'une révision des recommandations. Cette répétition d'une mise au point est rendue nécessaire par le constant et rapide progrès des connaissances scientifiques en général, et de leur répercussion sur la diffusion des risques d'exposition à des rayonnements ionisants. La découverte de la radioactivité artificielle et l'acquisition de l'énergie nucléaire ont fait passer le problème de la protection du domaine de l'individu à celui de la société. Les difficultés et les perspectives nouvelles qui en résultent vont vous être présentées par le président M. K.Z. Morgan. 